\title{
Tumor Residual Pós-Quimioterapia Neoadjuvante para Câncer de Mama: Impacto sobre o Tratamento Cirúrgico Conservador
}

\author{
Residual Tumor after Neoadjuvant Chemotherapy for Breast Cancer: Impact on Conservative \\ Surgical Treatment
}

Edison Mantovani Barbosa, Nídia Fabrega Donoso, Cynthia Aparecida Bueno T. Osório, Eloá Muniz F. Alves, Fátima Cristina Waldvogel, Célia Tosello Oliveira, João Carlos Sampaio Góes.

\begin{abstract}
RESUMO
Objetivo: analisar as alterações histopatológicas provocadas pela ação da quimioterapia neoadjuvante (fluoracil, epirrubicina e ciclofosfamida; FEC - 4 ciclos) na área tumoral, no tecido mamário adjacente e nos linfonodos homolaterais, em peças cirúrgicas obtidas de pacientes portadoras de carcinomas primários da mama.

Método: estudo histológico detalhado de 30 peças cirúrgicas obtidas por mastectomia radical (Patey) de pacientes portadoras de carcinomas primários da mama, previamente submetidas a esse tipo de terapêutica sistêmica.

Resultados: observamos regressão tumoral, de grau variável, em todas as peças analisadas. Esta regressão ocorreu de forma irregular, restando inúmeros focos refratários na área ocupada pelo tumor primário. Observamos focos celulares resistentes independentes do tumor primário no tecido mamário. Detalhamos outros achados histopatológicos decorrentes da ação quimioterápica nos tecidos tumoral e mamário, como calcificações e fibrose, e nos linfonodos axilares homolaterais.

Conclusão: concluímos que a ação da quimioterapia neoadjuvante não é uniforme, restando focos tumorais refratários, tanto na área do tumor inicial, quanto à distância. A regressão do tumor independe da resposta de regressão dos linfonodos axilares metastáticos. A utilização da cirurgia conservadora pós-quimioterapia neoadjuvante (FEC) deve ser evitada.
\end{abstract}

PALAVRAS-CHAVE: Mama: câncer-tratamento. Quimioterapia. Cirurgia.

\section{Introdução}

A quimioterapia neoadjuvante (QTNA), também denominada quimioterapia primária, préoperatória ou de indução, foi introduzida no arsenal terapêutico do câncer de mama há cerca de 20 anos, com o objetivo de se conseguir uma redução

Instituto Brasileiro de Controle do Câncer, São Paulo, SP. Correspondência:

Edison Mantovani Barbosa

Av. Alcântara Machado, 2576

03102-002 - São Paulo - SP da massa tumoral em tumores localmente avançados e permitir a utilização de tratamentos cirúrgicos e radioterápicos mais eficientes ${ }^{22}$.

A idéia de sua utilização originou-se das observações da melhora do controle local com a utilização da quimioterapia adjuvante. Nos poucos trabalhos clínicos randomizados publicados constatou-se uma diminuição na probabilidade de recorrência e aumento na sobrevida das pacientes portadoras de câncer de mama localmente avançado submetidas a este tipo de tratamento. Isto se deveu à rápida diminuição do volume tumoral, conseguida 
em alguns casos, facilitando o emprego dos tratamentos cirúrgico e/ou radioterápico. Muitas pacientes tratadas desta maneira alcançaram reduções de mais de $50 \%$, tanto da massa do tumor primário, quanto da linfoadenopatia metastática regional, e entre 10 e $20 \%$ do total alcançam resposta clínica completa ${ }^{16,12,10}$.

Mais recentemente, os excelentes resultados alcançados com o emprego do tratamento cirúrgico conservador para tumores de até $2,0 \mathrm{~cm}$ abriram importantes perspectivas de sua aplicação em tumores localmente avançados, cujas massas tumorais sofressem reduções substanciais com o uso da quimioterapia neoadjuvante ${ }^{7,15,25}$.

Neste sentido, vários estudos têm sido publicados para documentar a eficácia da atuação da QTNA na redução do tumor primário, assim como das metástases existentes. No entanto, as respostas obtidas diferem entre os autores, independente do tipo de associação de drogas e do tempo utilizado. Isto se deve à heterogeneidade do comportamento biológico das células tumorais, o que provoca o aparecimento de fenótipos, espontâneos ou induzidos, de resistência aos quimioterápicos ${ }^{2}$, 13, 19, 21.

A eficiência do tratamento cirúrgico conservador após a indução quimioterápica tem sido demonstrada em estudos mais recentes ${ }^{5,8,24}$. Entretanto, a utilização dessa forma de tratamento tem despertado importantes questionamentos nos seus praticantes, uma vez que as avaliações clínicoradiológicas empregadas para medir tanto o tamanho tumoral quanto sua regressão após o tratamento sistêmico não se correlacionam.

Também não existe critério para selecionar qual padrão de regressão é indicado para este ou aquele tipo de cirurgia conservadora e qual a extensão deste procedimento. Além disso, a literatura é muito pobre em relação ao detalhamento das modificações histopatológicas, decorrentes da ação dos quimioterápicos, nos tecidos tumorais e adjacentes ${ }^{23}$.

Com objetivo de estudar a persistência de tecido neoplásico refratário na glândula mamária e as alterações provocadas pela ação da associação fluoracil, epirrubicina e ciclofosfamida $(\mathrm{FEC})$ na área tumoral, no tecido mamário adjacente e nos linfonodos axilares homolaterais, realizamos este estudo envolvendo uma série de 30 peças cirúrgicas obtidas em pacientes portadoras de câncer primário da mama, submetidas a este tipo de QTNA.

\section{Material e Métodos}

O material estudado consiste de 30 peças cirúrgicas obtidas por mastectomia radical modificada (cirurgia de Patey), em pacientes do sexo feminino portadoras de carcinomas primários de mama, estádios clínicos T2 e T3. Estas pacientes haviam sido anteriormente submetidas a quimioterapia neoadjuvante, com quatro ciclos de FEC nas seguintes doses: fluoracil - $500 \mathrm{mg} / \mathrm{m}^{2}$; epirrubicina - $50 \mathrm{mg} / \mathrm{m}^{2}$ e ciclofosfamida - $500 \mathrm{mg} / \mathrm{m}^{2}$.

Todos os casos tiveram o diagnóstico inicial estabelecido por meio de punção aspirativa com agulha fina (PAAF). Consideramos apenas os tumores histologicamente classificados como ductal invasivo, ao exame histopatológico.

Os tumores foram medidos clínica e radiologicamente na fase prévia ao tratamento. Após a QTNA as pacientes foram submetidas a novo exame clínico e mamográfico que, comparado com o primeiro, determinou o grau de redução da massa tumoral.

As peças cirúrgicas obtidas por mastectomia foram congeladas à temperatura média de $-18^{\circ} \mathrm{C}$, sem fixação prévia. Posteriormente, foram parcialmente descongeladas e submetidas a cortes seqüenciais transversais com $1,0 \mathrm{~cm}$ de espessura. As fatias foram numeradas da axila para a região medial e o produto do esvaziamento axilar foi separado, para posterior análise (Figura 1). Foram escolhidas 3 ou 4 fatias mais representativas do tumor residual macroscópico. Estas fatias foram copiadas em desenhos esquemáticos (Figura 2).

Em seqüência foram feitos cortes transversais de $1,0 \mathrm{~cm}$ de largura em toda a extensão das fatias. O corte que apresentou a porção mais representativa do tumor residual macroscópico foi designado como " $\mathrm{C}$ " (central) e os demais localizados à sua esquerda e à sua direita foram designados E1, E2 ....... En e D1, D2 ... Dn, respectivamente (Figura 3). Estes cortes foram, posteriormente, fixados em formol e submetidos à técnica de inclusão em parafina, de forma manual, sendo obtidos blocos correspondentes a cada corte, devidamente identificados quanto à fatia pertencente e à sua posição na peça. Os cortes de tecido foram corados pelo método de $\mathrm{H}-\mathrm{E}$ e examinados em microscópico óptico de luz.

O patologista observador, de posse do desenho das fatias em tamanho natural, analisou cada corte e localizou no desenho a posição microscópica real do tumor residual, sendo este totalmente mapeado. Foram designadas as áreas contendo tumor infiltrativo, tumor in situ, êmbolos tumorais linfáticos, além das alterações funcionais e proliferativas associadas no parênquima mamário adjacente. Foram também identificadas todas as alterações celulares tumorais e do estroma, supostamente conseqüentes ao tratamento quimioterápico. O restante da peça cirúrgica foi estudado de acordo com o protocolo macro e microscópico de estudo de peças cirúrgicas da mama, utilizado em nosso serviço de anatomia patológica. 


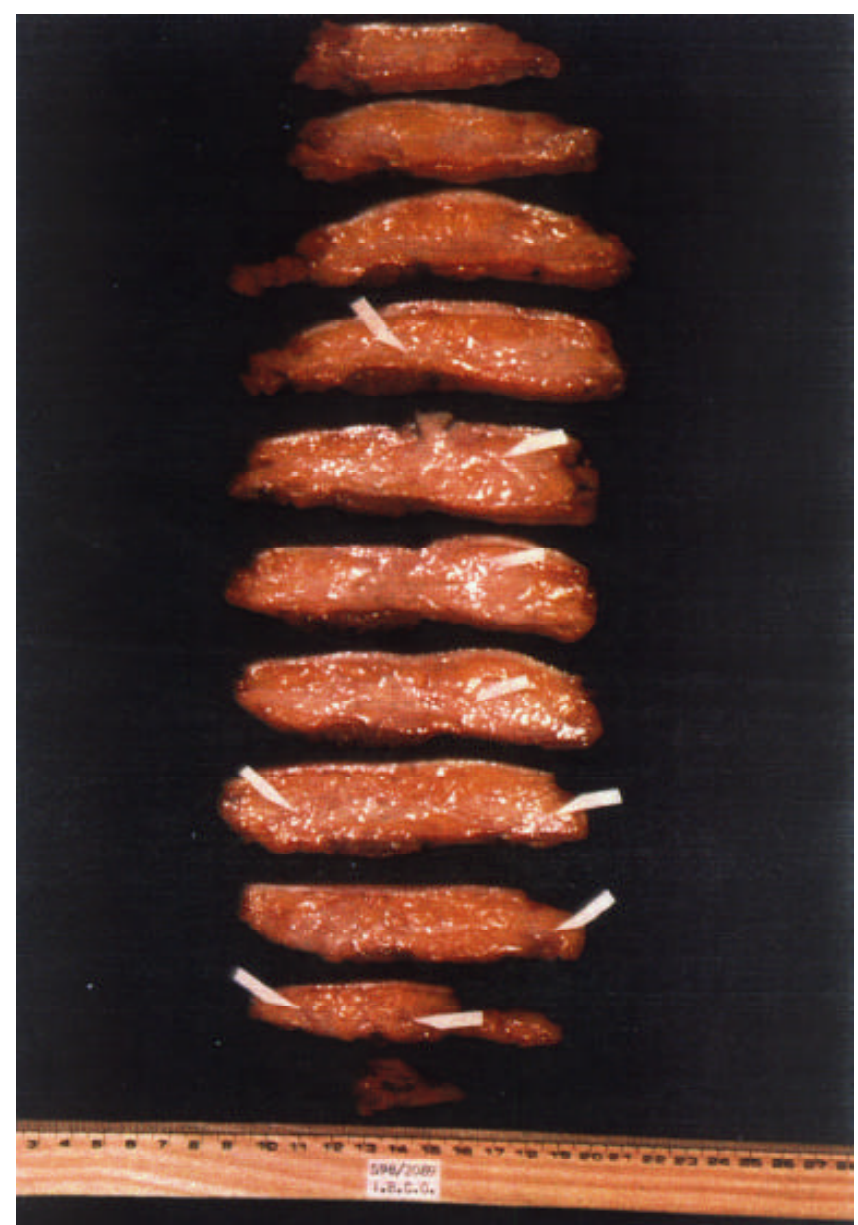

Figura 1 - Cortes de peça cirúrgica em fatias transversais seqüenciais da axila para região medial.

6a. Fatia

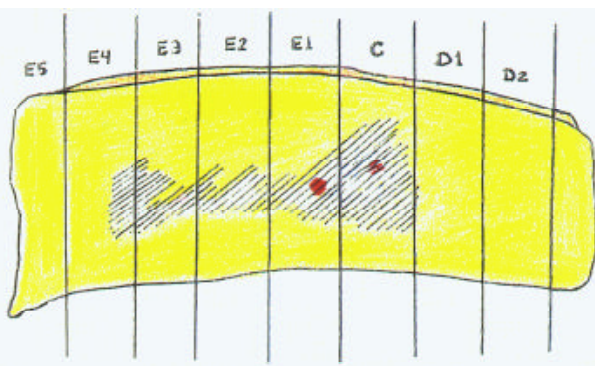

5a. Fatia

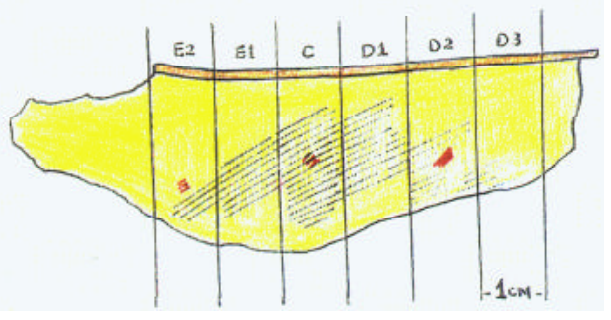

Figura 2 - Desenho esquemático de fatias selecionadas representativas do tumor macroscópico residual pós-quimioterapia e cortes seqüenciais submetidos a estudo histológico.

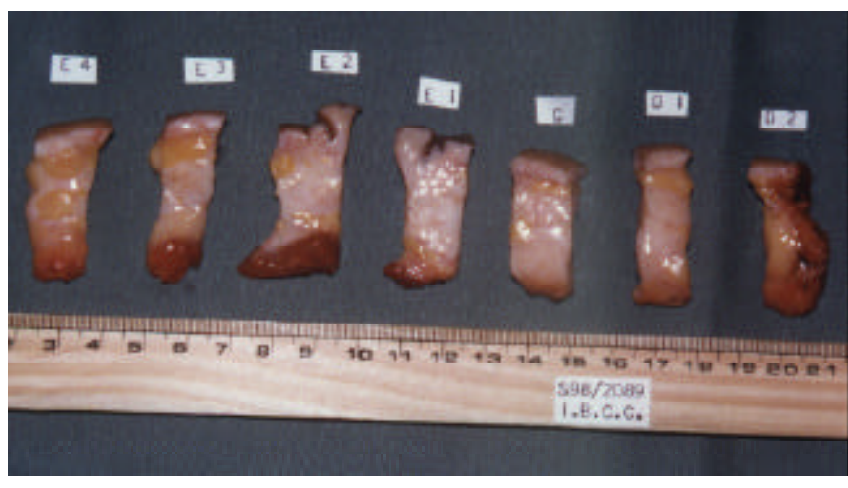

Figura 3 - Cortes transversais de fatia. " $\mathrm{C}$ " (central): maior representatividade tumora macroscópica.

\section{Resultados}

Em todas as peças estudadas observamos algum grau de regressão do volume da massa tumoral inicial, após a utilização da quimioterapia neoadjuvante. Tal regressão ocorreu de forma irregular, não obedecendo ao caráter centrípeto imaginado. Inúmeros focos tumorais residuais, isolados ou confluentes, foram observados circunjacentes às áreas de desaparecimento do tumor, representadas por fibrose hialina (desmoplasia), edema e necrose.

A presença do carcinoma intraductal associado ao invasivo foi observada em 18 casos (60,0\%); em 9 destes o padrão comedocarcinoma estava associado, em 2 outros o padrão cribriforme e, em mais 3 casos independentes, os padrões papilifero, sólido e micropapilar.

Em 2 casos, cujos tumores ao exame clínico mediam $5,0 \mathrm{~cm}$ cada, observamos a regressão quase total da massa tumoral, restando em um deles três microfocos de carcinoma invasivo esparsos, detectados apenas ao exame microscópico. No outro, constatamos, em uma extensão de $1,8 \mathrm{~cm}$, abundante degeneração celular e raras células neoplásicas viáveis.

Observamos embolias tumorais linfáticas em 22 casos $(73,3 \%)$. As áreas de necrose e fibrose foram achados freqüentes e na maioria abundantes, dissociando áreas de tumor microscópico, na sua adjacência ou em raros focos distantes da massa tumoral residual (Figuras 4 e 5).

Microcalcificações foram observadas em 21 casos $(70,0 \%)$ e em 4 destes elas não haviam sido detectadas pelos exames mamográficos prévios. Áreas de hiperplasia epitelial tipica e/ou atípica também foram observadas com bastante freqüência (11 casos: $36,6 \%$ ), ao passo que a metaplasia escamosa foi um achado esporádico. 


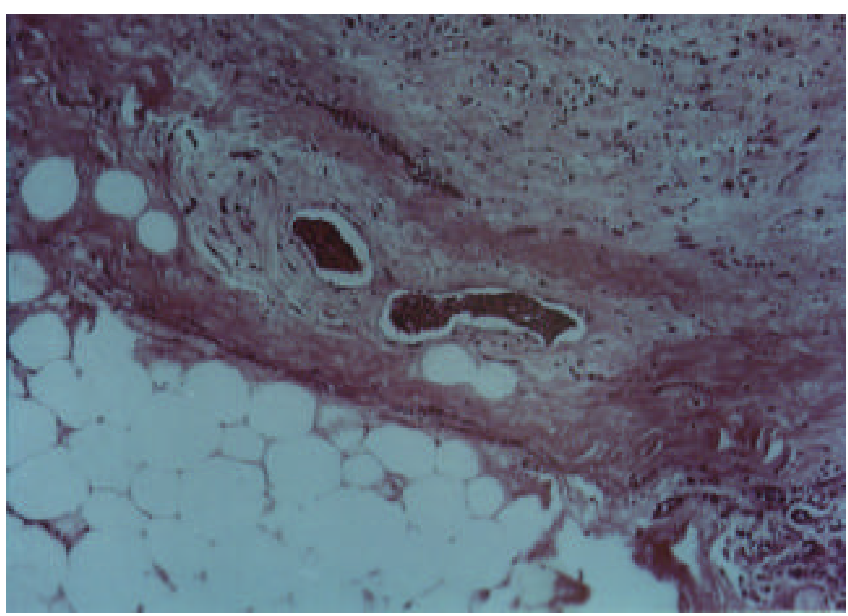

Figura 4 - Êmbolos linfáticos tumorais (lupa).

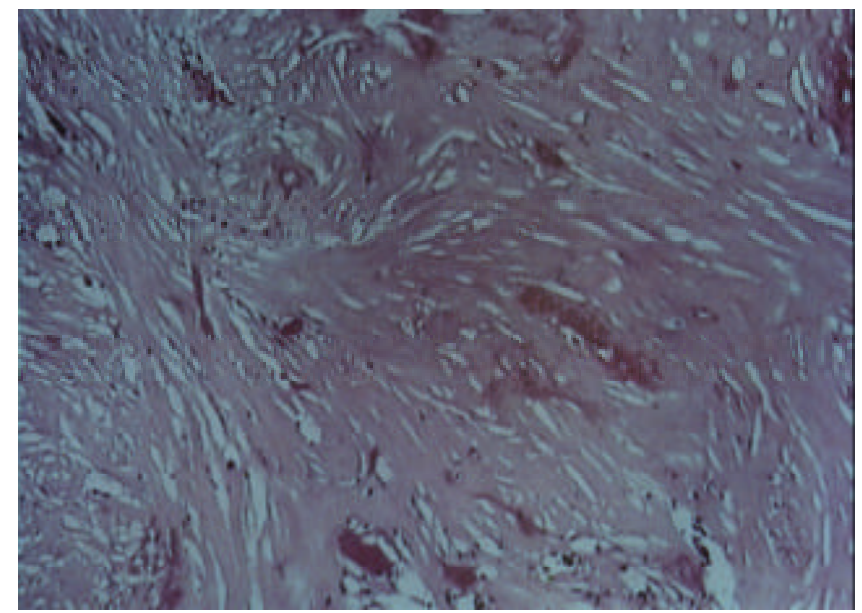

Figura 5 - Extensas áreas de fibrose e hialinização intersticial na região do tumor (lupa).

O infiltrado linfocitário peritumoral esteve presente em 19 casos (63,3\%), em graus variados, sendo intenso em apenas 4. Outros achados anátomo-patológicos estavam presentes na maioria dos casos, isolados ou associados, tais como: alterações fibrocísticas, adenose, adenose esclerosante, metaplasia apócrina, ectasia ductal, fibroadenomatose, papiloma e papilomatose.

As alterações citológicas observadas nas células neoplásicas foram evidentes somente em alguns casos e consistiram basicamente de alterações nucleares. Estas alterações foram caracterizadas por importante pleomorfismo, padrão granuloso de cromatina ou hipercromatismo e nucléolo proeminente.

Em um dos casos observamos intensa dissociação intersticial intratumoral decorrente do aumento de histiócitos. Uma intensa fibrose intersticial foi observada em 50\% dos casos, provocando a diminuição da quantidade do componente epitelial nestas regiões, devido sua permeação.

Em relação aos linfonodos axilares, 17 casos $(77,3 \%)$ apresentaram metástases. Em 5 destes casos $(22,7 \%)$ foram detectados até 3 glânglios comprometidos; nos demais, mais de 4 gânglios estavam envolvidos. Em um caso, pudemos observar intensa quantidade de necrose linfonodal sem evidências de células neoplásicas residuais, sugerindo uma importante regressão do processo metastático.

Os casos que apresentaram linfonodos axilares livres de comprometimento metastático ao exame histológico correlacionaram-se com tumores residuais de até $3,0 \mathrm{~cm}$, ao passo que o grupo de tumores residuais maiores se correlacionou com a positividade metastática linfonodal, mas não com o número de linfonodos envolvidos.

\section{Discussão}

Este estudo nos permitiu identificar uma série de alterações histológicas decorrentes do uso do esquema de quimioterapia neoadjuvante adotado (FEC - 4 ciclos) e concluir que as drogas utilizadas atuaram de maneira irregular e aleatória na regressão da massa tumoral. Percebemos inúmeros focos tumorais residuais, isolados ou confluentes, permeados por fibrose, edema e/ou necrose. Este achado é pouco referido na literatura e é a nosso ver muito importante, pois poderá estar diretamente relacionado ao percentual de recidiva local decorrente do emprego das cirurgias conservadoras.

Ao compararmos os estudos publicados envolvendo a utilização da QTNA em tumores localmente avançados, pudemos constatar diferentes esquemas, períodos de utilização e tumores tratados, resultando em importantes diferenças nos resultados encontrados $^{3,6}, 16,18,20,22,23$. No que se refere à irregularidade da resposta histológica de regressão da massa tumoral, vale ressaltar que os trabalhos publicados não fazem referência a este fato ${ }^{23}$.

Outra observação relevante para análise das taxas de resposta de regressão tumoral é a atenção e o detalhamento adotado pelo patologista durante o exame das peças operatórias, visto que focos microscópicos tumorais residuais podem não ser detectados, alterando significativamente os resultados obtidos. Reforçamos, com este estudo, nossas observações da falta de correlação entre os exames clínico, radiológico e histológico de extensão e regressão tumoral, pois não há qualquer possibilidade de se avaliar, com precisão, a extensão e a regressão da massa tumoral partindo unicamente dos exames clínico e mamográfico.

Alguns fatores contribuem para estes fatos: na 
avaliação clínica do tumor levamos em consideração a influência dos tecidos peritumorais, que nem sempre estão envolvidos pela neoplasia e são considerados na medida do diâmetro tumoral. Além disso, os freqüentes prolongamentos neoplásicos e focos isolados não podem ser avaliados por este método. Da mesma forma, não se consegue, pela análise do exame mamográfico, estabelecer com acuidade as áreas de extensão e regressão da massa tumoral, devido à substituição do tecido tumoral por fibrose, que apresenta a mesma densidade radiológica do tecido neoplásico.

A correlação entre resposta clínica e evidência de regressão histológica tem sido controversa na literatura. Por exemplo, Brifford et al. ${ }^{4}$ relatam uma correlação significante entre ambos, ao passo que Morrow et al. ${ }^{17}$ e Sharkey et al. ${ }^{22}$ compartilham a observação da falta de correlação, que também é defendida por nós.

Ao analisarmos as medidas de regressão tumoral obtidas somente pelo exame clínico, encontramos $60 \%$ de resposta parcial e $10 \%$ de resposta total. Esta mesma avaliação realizada pelo exame histopatológico evidenciou que em todos os nossos casos havia alterações teciduais compativeis com algum grau de regressão e em apenas 2 casos $(6,6 \%)$ encontramos regressão praticamente total da massa tumoral. Também neste tópico os trabalhos são controversos. Autores como Fisher et al. ${ }^{8}$, Bonnadonna et al. ${ }^{2}$ e Morrow et al. ${ }^{17}$ encontraram taxas de regressão total ao exame histológico ao redor de $5 \%$, ao passo que para Anderson et al. ${ }^{1}$ esta taxa alcançou $17 \%$.

Nosso achado de carcinomas infiltrativos residuais associados a carcinomas intraductais $(60,0 \%)$, comprometimento metastático linfonodal $(73,3 \%)$ e de embolia linfática $(73,3 \%)$ são compativeis com a freqüência relatada na literatura, em estudos cujos tumores apresentavam tamanhos semelhantes aos nossos ${ }^{8,9,11,14}$.

As microcalcificações observadas em 21 casos $(70,0 \%)$ foram detectadas tanto no tecido tumoral residual, como no parênquima mamário adjacente ao tumor. As alterações nucleares já referidas foram observadas de forma uniforme, tanto no tumor infiltrativo residual, como no componente ductal in situ e nos linfonodos axilares metastáticos. A fibrose foi freqüentemente observada no parênquima mamário adjacente e no tumor residual, sendo encontrada de forma mais acentuada em pacientes acima dos 50 anos (média 52) e menos acentuada em pacientes abaixo de 50 anos (média 47), contrariamente ao que Sharkey et al. ${ }^{22}$ observaram em seu estudo. Encontramos, ainda, embora em quantidade discreta, alterações citológicas no tecido não-neoplásico.

Quanto ao estudo dos linfonodos axilares, pudemos observar que aqueles não comprometidos pela neoplasia apresentavam aumento de evidência de depleção linfocitária e linfadenite de padrão sinusoidal.

Em resumo, este estudo nos permitiu chegar às seguintes conclusões: a ação do quimioterápico se faz predominantemente nas células neoplásicas e poucas alterações são observadas no tecido mamário não-neoplásico; inúmeros focos residuais são observados na área correspondente ao tumor inicial e no tecido mamário adjacente, sendo impossivel a sua avaliação clínica e radiológica; além disso, a resposta de regressão do tumor não está correlacionada com a resposta de regressão dos linfonodos axilares metastáticos.

Portanto, a utilização da cirurgia conservadora no carcinoma avançado de mama, que responde adequadamente à indução quimioterápica, deve ser lastreada em melhores conhecimentos a respeito da sua biologia evolutiva. Achamos que esta prática não deve ter utilidade rotineira, até que as seguintes dúvidas sejam esclarecidas: quais os critérios de seleção de regressão tumoral que permitem a indicação segura da cirurgia conservadora? a cirurgia conservadora e a radioterapia complementar são eficientes para o controle do tecido tumoral residual? qual o impacto deste tipo de tratamento no período livre de doença e na sobrevida?

\section{SUMMARY}

Purpose: analysis of histopathologic alterations caused by neoadjuvant chemotherapy (fluorouracil, epirubicine, cyclophosphamide; FEC - 4 cycles) at the tumor site, adjacent mammary tissue and homolateral lymph nodes, as observed in sections of patients with primary breast carcinomas.

Method: histological studies performed on 30 surgical sections obtained from radical mastectomy (Patey) of patients with primary breast carcinomas, who underwent prior neoadjuvant systemic therapy.

Results: all sections showed tumor regression with variable intensity. This regression occurred irregularly, several refractory tumor cells remaining at the primary tumor site. Resistant tumor cells, independent of the primary tumor, were found in mammary tissue. Other histopathological findings, resulting from chemotherapy in tumoral and mammary tissues, such as calcifications and fibrosis, and in axillary homolateral lymph nodes were obtained.

Conclusion: the effect of neoadjuvant chemotherapy is not uniform, refratory tumor cells remaining not only at primary tumor site, but also in distant regions. Furthermore, we found no correlation between the regression of the tumor and the axillary metastatic lymph nodes. Thus, a conservative surgery after neoadjuvant chemotherapy (FEC) should be avoided.

KEY WORDS: Breast cancer: treatment. Chemotherapy. Surgery. 


\section{Referências}

1. Anderson EDC, Forrest APM, Hawkins RA, Anderson TJ, Leonard RC, Chetty U. Primary systemic therapy for operable breast cancer. $\mathrm{Br} \mathrm{J}$ Cancer 1991; 63: 561-5.

2. Bonadonna G, Veronesi U, Brambilla C, Ferrari L, Luini A, Greco M, et al. Primary chemotherapy to avoid mastectomy in tumor with diameters of three centimeters or more. J Natl Cancer Inst 1990; 82: $1539-45$.

3. Bonadonna G, Veronesi U, Brambilla G, Ferrari L, Passoni P, Coopmans YGF, et al. Primary chemotherapy for resectable breast cancer. Recents Results Cancer Res 1993; 127:113-7.

4. Brifford M, Spyratos F, Tubiana-Hulin M, Pallud C, Mayras C, Filleul A, et al. Sequential cytopunctures during preoperative chemotherapy for primary breast carcinoma. Cytomorphologic changes, initial tumor plody, and tumor regression. Cancer 1989; 63: 630-5.

5. Calais G, Berger C, Descamps P, Chapet S, ReynaudBougnoux A, Body G, et al. Conservative treatment feasibility with induction chemotherapy, surgery, and radiotherapy for patients with breast carcinoma larger than $3 \mathrm{~cm}$. Cancer 1994; 74: 1283-8.

6. Casper ES, Guidera CA, Bosl GJ, Hayest B, Kaufman RJ, Shurgot B, et al. Combined modality treatment of locally advanced breast cancer. Adjuvant combination chemotherapy with and without doxorubicin. Breast Cancer Res Treat 1987; 9: 39-44.

7. De Lena M, Varini M, Zucali R, Rovini D, Viganotti G, Valagussa $P$, et al. Multimodal treatment for locally advances breast cancer: Results of chemotherapyradiotherapy versus chemotherapy-surgery. Cancer Clin Trials $1981 ; 4: 229-36$.

8. Fisher B, Brown A, Mamounas E, Wieand S, Robidoux A, Marcolesi RG, et al. Effect preoperative chemotherapy on local-regional disease in women with operable breast cancer. Findings from National Surgical Adjuvant Breast and Bowel Project B-18. J Clin Oncol 1997; 15: 2483-93.

9. Fowble B, Solin LJ, Schultz DJ, Rubenstein J, Goodman RL, Breast recurrence following conservative surgery and radiation: Patterns of failure, prognosis, and pathologic findings from mastectomy specimens with implications for treatment. Int $\mathrm{J}$ Radiat Oncol Biol Phys. 1990; 19: 833-42.

10.Harris JR, Morrow M, Bonadonna G, Cancer of the breast. In: De Vita VT Jr, Hellman S. Rosenberg AS, editores. Cancer: Principles \& Practice of Oncology. 4th ed. Philadelphia PA: JB Lippincott Co; 1993. p.1264-1332.

11.Hery M, Namer M, Moro M, Boublil JL, Lallanne C. Conservative treatment (chemotherapy / radiotherapy) of locally advanced breast cancer. Cancer 1986; 57:1744-9.

12.Hortobagy, G. N. Multidisciplinary management of advanced primary and metastatic breast cancer. Cancer 1994; 74: 416-23.

13.Hortobagyi GN, Blumenschein GR, Spanos W, Montague ED, Buzdar AU, Yap HY, Scheel F. Multimodal treatment of locoregionally advanced breast cancer. Cancer 1983; 51: 763-8.
14.Jacquillat C, Weil M, Baillet F, Borel C, Aureclerc G, De Maublanc MA, et al. Results of neo-adjuvant chemotherapy and radiation therapy in the breastconserving treatment of 250 patients with all stages of infiltrative breast cancer. Cancer 1990; 66:119-29.

15.Merajver SD, Weber BL, Cody R, Zhang D, Straw-Derman $\mathrm{M}$, Calzone KA, et al. Breast conservation and prolonged chemotherapy for locally advanced breast cancer: The University of Michigan experience. J Clin Oncol 1997; 15: 2873-81.

16.Morrel LE, Lee YJ, Hurley J, Arias M, Mies C, Richman $\mathrm{SP}$, et al. A phase II trial of neoadjuvant methotrexate, vinblastine, doxorubicin, and cisplatin in the treatment of patients with locally advanced breast carcinoma. Cancer 1998; 82: 503-11.

17. Morrow M, Braverman A, Thelmo W, Sohn CK, Sand J, Mora M, et al. Multimodal therapy for locally advanced breast cancer. Arch Surg 1986; 121: 1291-3.

18.Pierce LJ, Lippman M, Ben-Baruch N, Swain S, O'Shanghnessy J, BaderJL, et al. The effect of systemic therapy on local-regional control in locally advanced breast cancer. Int $\mathrm{J}$ Radiat Oncol Biol Phys 1992; 23:949-60.

19.Powles TJ, Hickish TA, Makris A, Ashley SE, O`Brien $\mathrm{ME}$, Tidy UA, et al. Randomized trial of chemoendocrine therapy started before or after surgery for treatment of primary breast cancer. J Clin Oncol 1995; 13: 547-52.

20.Scholl SM, Fourquet A, Asselain B, Pierga JY, Vilcoq JR, Durand JC, et al. Neoadjuvant versus adjuvant chemotherapy in premenopausal patients with tumors considered too large for breast conserving surgery: preliminary results of a randomized trial: 56 . Eur J Cancer 1994; 30 A:645-52.

21.Schwartz GF, Birchansky CA, Komarnicky LT, Mansfield CM, Cantor RI, Biermann WA, et al. Induction chemotheraphy followed by breast conservation for locally advanced carcinoma of the breast. Cancer 1994; 73:362-9.

22.Sharkey FE, Addington SL, Fowler LJ, Page CP, Cruz AB. Effects of preoperative chemotherapy on the morphology of the resectable breast carcinoma Mod Pathol 1996; 9: 893-900.

23.Touboul E, Laurent B, Lefranc JP, Blondon J, Deniaud E, Mammar H, et al. Possibility of conservative local treatment after combined chemotherapy and preoperative irradiation for locally advanced noninflammatory breast cancer. Int J Radiat Oncol Biol Phys 1996; 34: 1919-28.

24.Veronesi U, Bonadonna G, Zurrida S, Galimberti V, Greco M, Brambilla C, et al. Conservation surgery after primary chemotherapy in large carcinomas of the breast. Ann Surg 1995; 222 612-18.

25.Veronesi U. New trends in breast cancer surgery. Recents Results Cancer Research 1989:115:197-202.

\section{Agradecimento}

Agradecemos aos Srs. Sebastião Cascaldo e Alexandre Jun Daniel Nakamae pela importante contribuição técnica no preparo do material analisado. 